\title{
DEVELOPMENT OF HARDWARE AROUND A SILICON MICROSTRIP DETECTOR WITH 512 ELEMENTS
}

\author{
Erik H.M. HEIJNE, Emilio HIGON *, Pierre JARRON and Leonardo ROSSI ** \\ CERN, Geneva, Switzerland
}

Paul BURGER

ENERTEC-Schlumberger, Lingolsheim, France

\author{
Wim GEERLINGS and Maarten RIJNSENT \\ Space Research Laboratory, Utrecht, The Netherlands
}

The silicon chip is mounted on a ceramic substrate and connected to the preamplifiers via kapton foils. A test bench is constructed, which enables measurement of reverse current and capacitance of all strips simultaneously.

\section{Introduction}

For an experiment aiming at the detection of heavy quark decays [1] in' the Omega $\left(\Omega^{\prime}\right)$ spectrometer at CERN, ten silicon microstrip detectors with 512 sensitive elements have been constructed, using the technology described by Kemmer [2]. To obtain a light and very compact construction of the detector telescope, a new design for the mounting has been made, using thin film ceramic plates with kapton connections. In order to check the large number of elements, a computerized detector test facility has been developed.

It has been possible to reduce both the size and the cost of the front-end amplifiers, so that they fit very well to the concept of the multi-element microstrip detector. These amplifiers are elsewhere described in detail by Jarron and Goyot [3]. The read-out of the channels is performed with an upgraded version of the existing $\Omega^{\prime}$ wire chamber electronics, which employs signal discrimination, followed by digital storage and multiplexing [4].

\section{The detector mounting}

The silicon microstrip detector, manufactured by Enertec (France), consists of $51250 \mu \mathrm{m}$ wide and 26 mm long strips. Above and below there are four "guard"

* On leave from Facultad Ciencas, Universidad de Valencia, Spain.

** On leave from Istituto Nazionale di Fisica Nucleare, Genoa, Italy. strips and bonding pads are staggered with $100 \mu \mathrm{m}$ pitch, alternatively at the left (odd strips) and the right (even strips). The overall size of the silicon chip is 30 $\mathrm{mm} \times 30 \mathrm{~mm} \times 0.3 \mathrm{~mm}$. It is glued with conductive silver epoxy in a hole in the ceramic substrate. Ultrasonic wedge bonding with $25 \mu \mathrm{m}$ thick aluminium wire connects each detector strip to a thin film lead on the ceramic.

The ceramic substrate, supplied by Balzers (Liechtenstein), has dimensions $101.6 \times 101.6 \times 0.6 \mathrm{~mm}^{3}$ and is covered on both sides with the usual sputtered thin resistive and conductive films. The conductive $\mathrm{Au}$ layer is electrolytically increased in thickness to $3 \mu \mathrm{m}$. A fan-out pattern is then etched, which ends in strips of $100 \mu \mathrm{m}$ width with a pitch of $320 \mu \mathrm{m}$, along the edge of the ceramic substrate.

A kapton foil of $50 \mu \mathrm{m}$ thickness, with a $17 \mu \mathrm{m}$ thick copper layer, carries the next stage of fan-out, and provides a flexible connection to the boxes with the preamplifiers, as is shown in fig. 1. Leads on the kapton foil make contact with the leads on the ceramic via a dot of conductive silver epoxy paste, which is deposited on the ceramic through "thick film" screen printing. The precise positioning of kapton and ceramic is carried out on a special $x-y$ table and once the patterns coincide, the kapton is clamped to the ceramic. The epoxy is then polymerized in an oven, at $90^{\circ} \mathrm{C}$.

Two detectors are mounted perpendicular to each other in a sandwich, as is shown in fig. 2. The overall width of such a sandwich is only $40 \mathrm{~mm}$, including the 1024 preamplifiers. Thanks to the kapton and the thin ceramic, the "open" area around the beam has a $13 \mathrm{~cm}$ diameter. The flat cables, which transmit the signals 


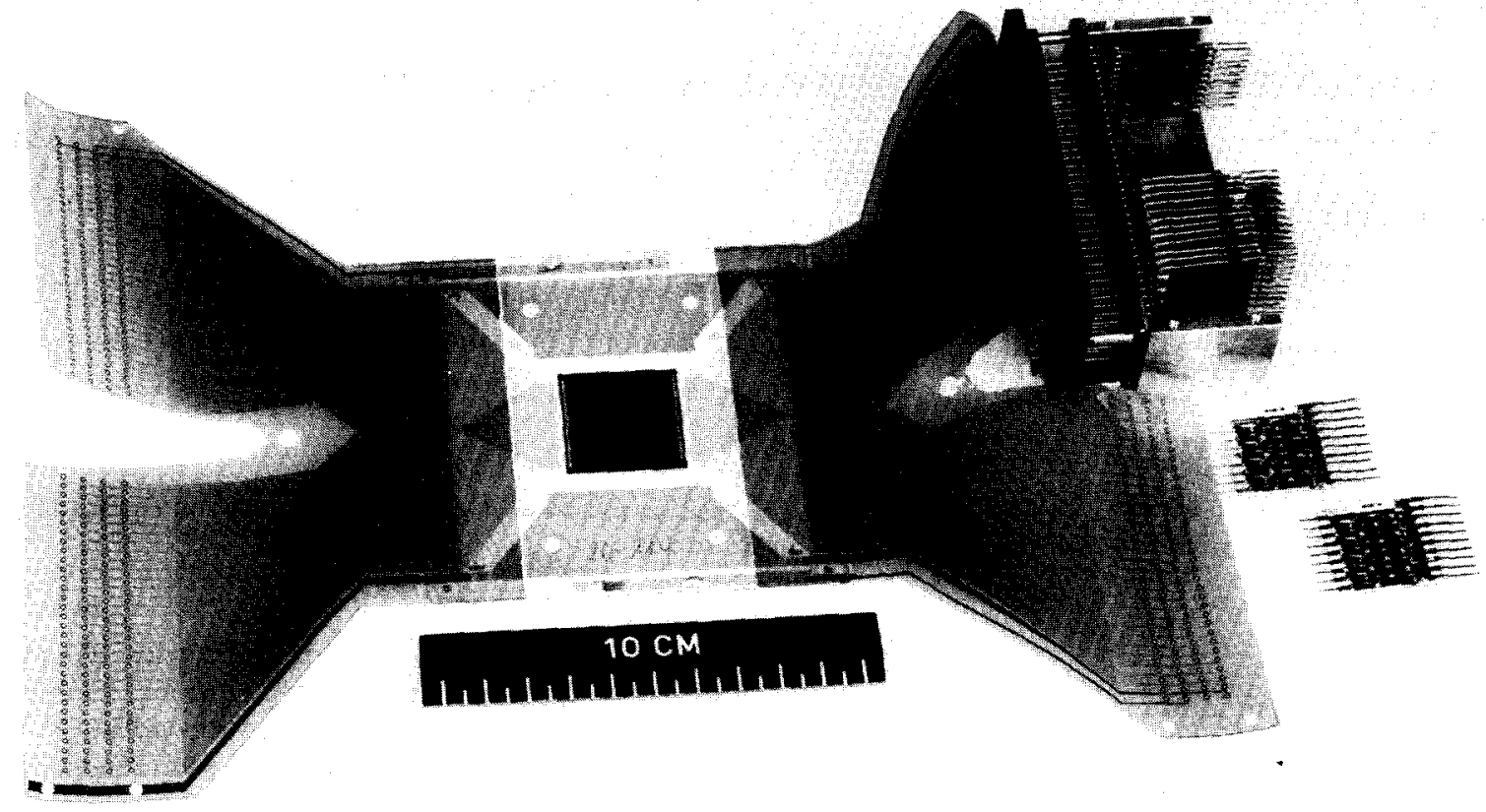

Fig. 1. Picture of the silicon chip, glued on a 4 inch $\times 4$ inch ceramic, with kapton foils connecting to one of the four amplifier boxes. The connector to the box requires only low insertion force. It has 128 pins for the signals and additional pins for ground and high voltage. The bias high voltage is carried to the rear side of the detector via bridges at the edge of the ceramic.

from the preamplifiers to the read-out system, constitute the largest fraction of the weight of the two-detector module.

\section{Detector test facility}

It is awkward to test a 512 strip detector by hand. An automatic system should provide the reverse diode currents of each strip, the capacitance of each strip to ground and the capacitance between strips. Of course it should enable the finding of unconnected strips and short circuits between adjacent strips.

Such a measurement system has been built using the LF 355 operational amplifier as a current to voltage converter. The strips are grounded via the input impedance $Z$ of the amplifiers and the reverse current $I_{\mathrm{r}}$ in each strip is measured as a voltage $V_{r}$, proportional to the feedback resistance $R_{\mathrm{f}}$. A schematic of the layout is given in fig. 3 and a picture of the test bench is shown in fig. 4. The output voltages are multiplexed, converted to digital and read via CAMAC into a CAVIAR computer. A computer program stores the values and produces various displays of the measurement results. The full current range is $1 \mu \mathrm{A}$ for a single strip with $0.5 \mathrm{nA}$ precision. An offset measurement at $0 \mathrm{~V}$ detector bias should precede this current measurement.

For the capacitance measurement, a radio frequency (RF) sinusoidal signal $V_{\mathrm{T}}$ of $0.5 \mathrm{~V}$, with a frequency $f=100 \mathrm{kHz}$, is applied at the rear side of the detector via a bias capacitor $C_{\mathrm{B}}$. This modulation of the reverse bias voltage creates a signal current $I_{\mathrm{c}}$ in the strip diodes,

$I_{\mathrm{c}}=V_{\mathrm{T}} /\left(Z+1 / \mathrm{j} 2 \pi f C_{\mathrm{s}}\right)=V_{\mathrm{T}} \mathrm{j} 2 \pi f C_{\mathrm{s}}$.

For small values of the diode strip capacitance $C_{\mathrm{s}}$, the input impedance $Z$ of the amplifier can be neglected (e.g. $Z \sim 500 \Omega$ for a feedback capacitance $C_{\mathrm{F}}=30 \mathrm{pF}$ and the second term $\sim 10^{6} \Omega$ for $C_{\mathrm{s}}=1 \mathrm{pF}$ ). The signal current $I_{\mathrm{c}}$ is amplified and it is found experimentally 


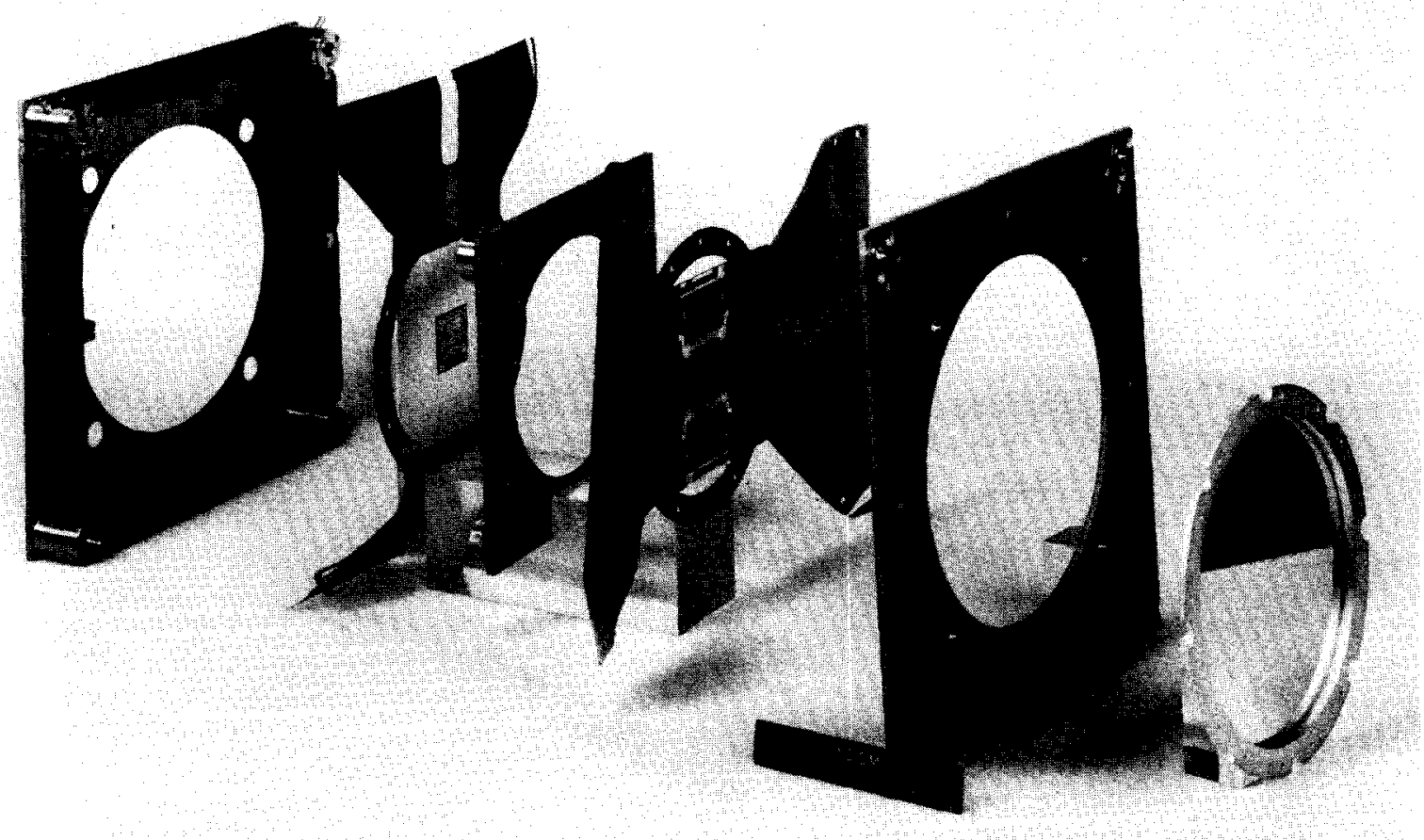

Fig. 2. Detectors are precisely mounted on stainless steel rings, such that the silicon chip is parallel to the ring. A horizontal and a vertical detector are mounted back to back with $8 \mathrm{~mm}$ interspace in a sandwich. Eight electronics boxes with a total of 1024 preamplifiers can surround the detectors.

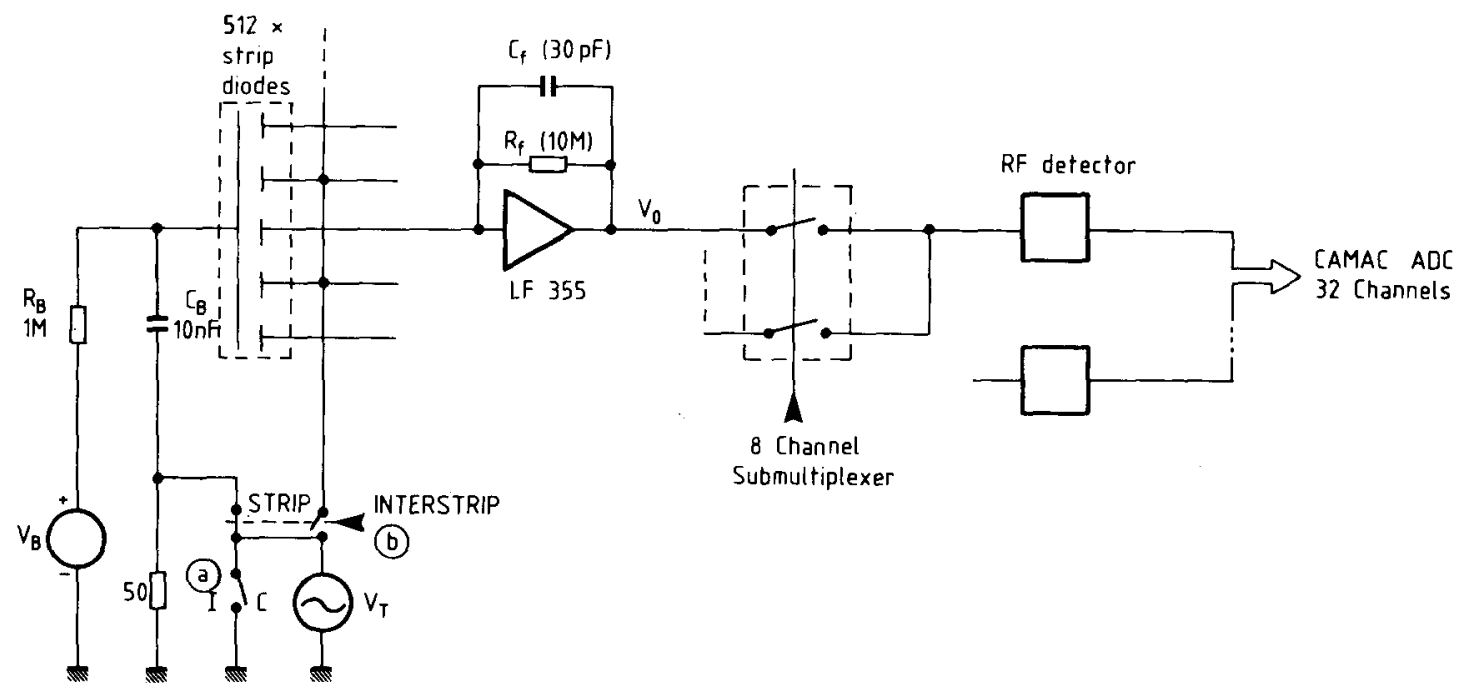

Fig. 3. Schematic of the testing system for silicon microstrip detectors with 512 strips. The reverse detector bias $V_{\mathrm{B}}$ is applied at the rear side of the detector. Each strip is connected to an operational amplifier, which operates as a current to voltage converter. If switch a is closed, the reverse current is measured, if it is open, the RF signal $V_{\mathrm{T}}$ is applied to the detector and an additional current is measured as a function of the strip diode capacitance. With switch $b$, the RF signal can be applied to the odd (or even) strips and in this case the interstrip capacitance can be measured. 


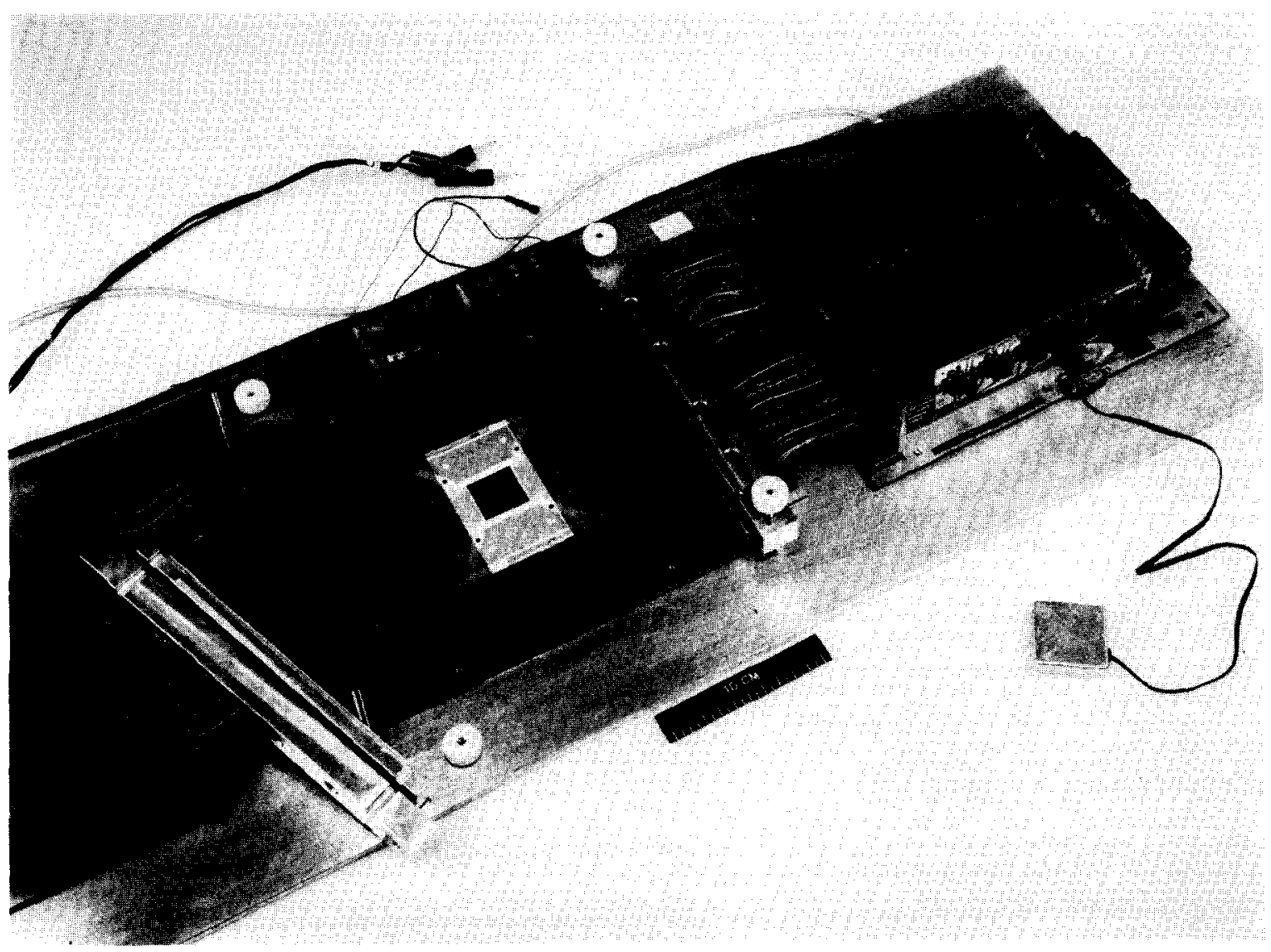

Fig. 4. Picture of the test bench. The detector is placed in the middle and connection is made by pressing the kapton foil on a corresponding printed circuit. The amplifiers are placed left (odd) and right (even strips). Each vertical card contains eight channels, with the submultiplexer and the RF detector. The CAMAC ADC (32 channels) and the CAVIAR computer are not shown.

that $0.18 \mathrm{~V} / \mathrm{pF}$ is added to the reverse current signal $V_{\mathrm{r}}$. To determine the capacitance of the strip, one therefore has to first measure the current-related part of the signal, at a given reverse bias voltage.

Any parasitic capacitance will appear in parallel to the input impedance $Z$ and can be neglected, as long as one measures a small capacitance. This constitutes an advantage of this method over a bridge-type measurement.

For the measurement of the interstrip capacitance, the RF signal $V_{\mathrm{T}}$ is applied on all even or all odd strips. The signal is then seen by the amplifiers on the other set of strips through the interstrip capacitances. Of course the capacitances to the strips above and below the measured strip are measured together at the same time. The precision of the capacitance measurements is 0.02 $\mathrm{pF}$.

In this test set-up, the unconnected strips can be found only in the capacitance measurement, because the reverse currents are often very low, so that the apparent absence of current should not be interpreted as a broken contact. A short circuit between adjacent strips gives rise to a large offset current $I_{0}$ at the inputs of both amplifiers, due to the offset voltage $V_{0}$. For example, for $Z=500 \Omega$, one finds $I_{0}=10 \mu \mathrm{A}$ for $V_{0}=5 \mathrm{mV}$.

Finally, the test bench is not only used to test detectors, but also to check the substrates of ceramic and kapton before mounting and bonding of the detector. In this check, the RF signal is applied via a uniformly metallized kapton foil, which is pressed against the open ends of the metal leads on the ceramic. The coupling capacitances obtained in this way are -0.3 $\mathrm{pF}$.

\section{Conclusion}

The size of silicon microstrip detectors and their number of elements are steadily increasing. Apart from the need for well-adapted electronics, the need for test- 
ing facilities and practical interconnection structures is also felt. With kapton foils, the risk of cabling errors is practically reduced to zero. A high density of connections is achieved (256 on $80 \mathrm{~mm}$ ) in a single layer construction. However, a special bench is needed to align the silicon chip and the kapton with respect to the ceramic substrate. The cost of the finished substrate is relatively high $(-800 \mathrm{SF})$, though still only a fraction of the total detector cost. It becomes desirable, therefore, to also test the detector chip before mounting, so that the substrate cost and also the cost of the bonding can be saved if the chip presents some flaws.

Many people have made essential contributions to this project, at CERN, in Utrecht and in Lingolsheim.
Their efforts are greatly appreciated. It is fair to mention especially $\mathrm{R}$. Pegaitaz, who forgot to take his holidays, G. Reiss who retired after this project and $A$. Menetrey, who made his come-back with this project.

\section{References}

[1] M. Adamovich et al., CERN/SPSC 81-18, SPSC/P159 and Addendum (Experiment WA71).

[2] J. Kemmer, these Proceedings, (Semiconductor Detectors '83), p. 89.

[3] P. Jarron and M. Goyot, these Proceedings, p. 156.

[4] F. Bourgeois had designed this system. 